Research Paper

\title{
Is pharmacologic treatment better than neural mobilization for cervicobrachial pain? A randomized clinical trial
}

\author{
César Calvo-Lobo1 ${ }^{1}$, Francisco Unda-Solano², Daniel López-López ${ }^{3 凶}$, Irene Sanz-Corbalán, Carlos \\ Romero-Morales ${ }^{5}$, Patricia Palomo-López ${ }^{6}$, Jesús Seco-Calvo7, David Rodríguez-Sanz ${ }^{5}$ \\ 1. Nursing and Physical Therapy Department, Institute of Biomedicine (IBIOMED), Universidad de León, Ponferrada, León, Spain. \\ 2. Interuniversity Degree in Physiotherapy UB-UdG / Grau en Fisioteràpia EUSES-UdG, Barcelona, Spain. \\ 3. Research, Health and Podiatry Unit, Department of Health Sciences, Faculty of Nursing and Podiatry, Universidade da Coruña, Spain. \\ 4. Faculty of Nursing, Physiotherapy and Podiatry. Universidad Complutense de Madrid, Spain \\ 5. European University. School of Sports Science. \\ 6. University Center of Plasencia, Faculty of Podiatry, Universidad de Extremadura, Spain. \\ 7. Institute of Biomedicine (IBIOMED), University of León, León (Spain). Researcher and Visiting Professor at the University of the Basque Country \\ (UPV/EHU), Spain
}

$\square$ Corresponding author: Daniel López López, Universidade da Coruña, Unidade de Investigación Saúde e Podoloxía, Departamento de Ciencias da Saúde, Campus Universitario de Esteiro s/n, 15403 Ferrol (España). Email: daniellopez@udc.es.

(c) Ivyspring International Publisher. This is an open access article distributed under the terms of the Creative Commons Attribution (CC BY-NC) license (https://creativecommons.org/licenses/by-nc/4.0/). See http://ivyspring.com/terms for full terms and conditions.

Received: 2017.10.27; Accepted: 2018.02.03; Published: 2018.03.08

\begin{abstract}
Purpose: This study aim was to compare the effectiveness of the median nerve neural mobilization (MNNM) and cervical lateral glide (CLG) intervention versus oral ibuprofen $(\mathrm{OI})$ in subjects who suffer cervicobrachial pain (CP).

Methods: This investigation was a, multicenter, blinded, randomized controlled clinical trial (NCT02595294; NCT02593721). A number of 105 individuals diagnosed with CP were enrolled in the study and treated in 2 different medical facilities from July to November 2015. Participants were recruited and randomly assigned into 3 groups of 35 subjects. Intervention groups received MNNM or CLG neurodynamic treatments, and the (active treatment) control group received an OI treatment for 6 weeks. Primary outcome was pain intensity reported through the Numeric Rating Scale for Pain (NRSP). Secondary outcomes were physical function involving the affected upper limb using the Quick DASH scale, and ipsilateral cervical rotation (ICR) using a cervical range of motion (CROM) device. Assessments were performed before and 1 hour after treatment for NRSP (baseline, 3 and 6 weeks) and CROM (baseline and 6 weeks), as well as only 1 assessment for Quick DASH (baseline and 6 weeks).

Results: Repeated-measures ANOVA intergroup statistically significant differences were shown for CP intensity $\left(F_{(2,72)}=22.343 ; P<.001 ; \mathrm{Eta}^{2}=0.383\right)$ and Quick DASH $\left(F_{(2,72)}=15.338 ; P<.001\right.$; $\left.\mathrm{Eta}^{2}=0.299\right)$, although not for $\operatorname{CROM}\left(\mathrm{F}_{(2.72)}=1.434 ; P=.245 ; \mathrm{Eta}^{2}=0.038\right)$. Indeed, Bonferroni's correction showed statistically significant differences for $C P$ intensity $(P<.01 ; 95 \% \mathrm{Cl}=0.22-3.26)$ and Quick DASH reduction $(P<.01 ; 95 \% \mathrm{Cl}=8.48-24.67)$ in favor of the Ol treatment at all measurement moments after baseline.

Conclusions: OI pharmacologic treatment may reduce pain intensity and disability with respect to neural mobilization (MNNM and CLG) in patients with CP during six weeks. Nevertheless, the non-existence of between-groups ROM differences and possible Ol adverse effects should be considered.
\end{abstract}

Key words: Neck; Non-steroidal anti-inflammatory agents; Musculoskeletal manipulations; Rehabilitation; Upper extremity. 


\section{Introduction}

Cervicobrachial pain (CP) is defined as the presence of neck pain that radiates or referrers to the upper limb that may be derived from neuropathic, pathomechanical, and degenerative disorders, as well as infections and systemic diseases [1-3]. Early descriptions involving $\mathrm{CP}$ can be found in the Edwin Smith medical papyrus dated 3700 BC [4], never the less, recent information regarding the incidence of this condition is not available. Most CP studies refer an incidence of 83 per every 100.000 individuals based on Radhakrishnan et al. [2] findings, accompanied by a 5 year prevalence of symptoms. 2016 up to date data reported by Gangavelli et al. [3] concludes that only $19.9 \%$ of CP cases are truly of neurogenic origin.

Gold standard diagnosis of $\mathrm{CP}$ is achieved by the presence of a positive correlation between clinical and radiographic pathological findings in a magnetic resonance imaging (MRI) procedure [5-8]. Other diagnostic methods for this condition are pathologic findings in nerve electro conduction evaluations and positive outcomes in orthopedic tests: Spurling, upper limb (ULT) and distraction test [9-11].

First line treatment of $\mathrm{CP}$ is done by a conservative pharmacological approach, employing non-steroidal antiinflammatory drugs (NSAIDs) such as oral ibuprofen (OI) (placed among the principal NSAIDs prescribed worldwide to treat pain and $\mathrm{CP}$ ) and specific physiotherapy techniques, meanwhile surgical procedures are should be reserved for cases of life- threatening co-morbidities or disabling pain [12-15].

Interesting data of over 6 studies concluded the presence of nerve trunk mechano-sensibility alterations of the cervicobrachial neural components during the onset of $\mathrm{CP}$. The presence and desired reversal of central sensitization are key elements to be considered when selecting a proper treatment method for this condition [16-21]. Specific physical therapy procedures designed to treat $\mathrm{CP}$ are believed to target these key elements that produce neuromechanical dysfunction and central sensitization. These series of procedures include manual orthopedic therapy, dry needling, myofascial release and neurodynamic maneuvers of cervical contralateral glide (CLG) as well as median nerve neural mobilization (MNNM) $[11,16,22,23]$.

Neurodynamic techniques of CLG and MNMM were originally developed by Butler et al., Coppieters et al., and Elvery-Hall as provocation test, and posteriorly evolved into treatment methods [24-26]. These techniques were designed to achieve CP relief through controlled mechanical stimulation of the median nerve and the brachial plexus. Although the entire set of underlying reasons for this pain reduction effect is not completely understood, it is assumed that prescribed mechanical stimulation of a nerve and its surrounding tissue may induce a variety of positive neuro-physiologic responses that improve pain threshold to stimuli, due to the activation of an inhibitory descending nervous system pathway. The positive effects derived from the application of MNNM and CLG that are linked to pain modulation are: changes on the viscoelastic properties of the nerve and local musculoskeletal tissue, indirect joint mobilization, intraneural pressure and edema reduction, dispersion of pro-inflammatory substances and an increase in nerve mobility $[17,27,28]$.

Both OI and neurodynamic treatments (MNNM and CLG) are believed to be effective in treating CP; nevertheless, this happens through extremely different physiologic pathways, and therefore, both treatments present vastly distinctive side effects. An OI treatment constitutes an oral intake of a drug, originally designed to control pain, fever and inflammation. OI hypoalgesic and anti-inflammatory effect is achieved by chemical inhibition of the COX enzymes that convert arachidonic acid to prostaglandin $\mathrm{H} 2$ (PGH2), which is then converted by other enzymes to several types of mediators of inflammation and pain $[18,29,30]$.

OI is capable of producing a vast quantity of side effects that can be severe in some patients even inside the limits of a regular over the counter doses. Therefore, OI may not be suitable for treatment in all types of subjects who suffer CP. Meanwhile the neurodynamic treatment for CP (MNNM and CLG) has no important side effect when applied properly, with the only exception of a temporary worsening of the subjects' symptomatology. This constitutes a very interesting point of comparative effectiveness between these two first line treatment alternatives of CP $[12,17,31]$. Additional, comparative randomized clinical trials (RCT) regarding MNNM, CLG and OI in $\mathrm{CP}$ without the mixed combination of other treatments are currently non-existent, which by itself is stand-alone challenge in regards to proper treatment selection for the practicing clinician who desires high quality evidence on the neurodynamic treatments (MNNM and CLG) level of effectiveness when compared to common over the counter pharmaceutical treatment for CP [29,32-36].

Based on the previously exposed rationale, the objective of the present study was to compare the pain intensity, functionality and cervical range of motion effectiveness of the MNNM and CLG neural mobilization treatments versus a pharmacological 
treatment with oral Ibuprofen (OI) in subjects with CP.

\section{Methods}

\section{Trial design}

This study was a controlled, experimental, randomized, multicenter, single-blind clinical trial that was performed to establish efficacy between treatments. The present investigation had 3 research arms (2 active experimental treatment arms and 1 active control arm) with a 1: 1: 1 allocation radius, conducted in Venezuela (in two locations). One of the study's co-authors (FUS, Venezuela) contacted with the centers, local physicians and physical therapists from Venezuela which were involved in the recruitment and outcome measurements. Furthermore, FUS was the responsible author to get the Ethics committee approval and organize the completion of the study. At all times participants and researchers were emphasized the need to maintain blinding. During the present study, the CONSORT and up to date World Medical Association's Declaration of Helsinki guidelines were followed. Valencia (Venezuela) Polyclinic Center Ethics Committee approved this study (code CE0072015, CE0072015-2). All subjects gave a written informed consent to participate in the present investigation. The individual of the Fig 1 and Fig 2 in this manuscript has given written informed consent to publish these case details. The trial was registered at ClinicalTrials.gov (NCT02595294, NCT02593721).

This study was based on a combination of 2 approved studies with a deviation of the sample size calculation from the original protocols (codes CE0072015 and CE0072015 of ethics committee approvals; and NCT02595294 and NCT02593721 number clinical trial registries) in order to include 3 groups in the study by means of a stratified randomization. These changes were performed after completion these original protocols and communicated to the ethics committee. The authors confirm that all ongoing and related trials for this drug/intervention are registered. Nevertheless, there was a delay in registering this study (October 2015, after enrolment of participants started). The trial was not prospectively registered, although the recruitment began immediately after the Ethics Committee approval date (July, 2015). In order to perform the recruitment associated to a doctoral process of the author FUS, the research process had to be adapted to the PhD schedule. Raw data of the demographic data and main outcomes measurements is available as S1 Raw data for all treatment groups. Subjects were assigned to 1 of the 3 groups (each group contained 35 subjects) using restricted block stratified randomization through a block computerized randomization software.

\section{Participants}

The study population was composed by subjects with medical diagnosis of CP. A total of 144 subjects were recruited and evaluated from July to November 2015. All necessary medical assessments including diagnosis and corroboration of pathological findings that were present in the MRI study and performed by a specialized physician [5-8]. A sample of 105 participants was considered suitable for recruitment, which were divided into three groups of 35 participants. The enrollment of subjects was performed by the specialized physician (internist medical doctor) according to the randomization scheme generated by the statistical analyst. Group "A" contained subjects treated with MNNM, group "B" contained subjects treated with OI and group "C" those participants treated with CLG. Inclusion criteria for participants were the following: Adults aged 18-45 years of both genders, who presented a signed informed consent to participate, a diagnosis of unilateral CP confirmed by MRI and Spurling, Distraction, and Upper Limb Orthopedic tests [5-11]. Exclusion criterions for participating subjects were: the presence NSAIDs intake contraindication, the use of any type of pain relief treatments at the current moment of enrollment, the presence of stenosis due to myelopathy, vertebral instability, cognitive impairment, pregnancy, kinesiophobia (Tampa Scale for Kinesiophobia (TSK-11) score > 34.04) [37], spinal cord or vertebral surgery, osteoporosis, infections, deformities or neoplasia in the medical record [29,32-36].

\section{Randomization and blinding}

The stratified randomization and allocation to trial group protocol were carried out through computer software randomized machine-printed cards. These cards were then placed inside a series of serial numbered non-translucid envelopes, which were completely sealed. The printed cards displayed an alphabetical letter that corresponded to one of the 3 groups. Randomization and allocation was designed by the data analyst.

The sealed envelopes were then handled to the internist medical doctor who delivered the envelopes to the candidates according to schedule. The internist medical doctor was blinded to the tested hypothesis and group randomization. Subject and physical therapist outcome assessor were blinded to the randomization, the group allocation, and the tested hypothesis; this blinding process was achieved by 
concealing the existence of other groups as well as the tested hypothesis. The subject and physical therapist outcome assessor were kept blinded after the assignment to intervention. The concealment of information between subjects and all the investigation members played a crucial role in the achievement of the study's blinding process [33].

\section{Main outcome}

$\mathrm{CP}$ intensity was measured through the numeric rating scale for pain (NRSP) on intervention sessions 1 (at baseline), 15 (at 3 weeks) and 30 (at 6 weeks), before and 1 hour after the application of the MNNM, DLC and OI treatments. CP intensity was considered the primary outcome (standard error of measure was equal to 1.02) [36,38]. The NRSP consists of a horizontal straight line of $11 \mathrm{~cm}$ subdivided in numbers which are equidistant from 0 to 10 , so that " 0 " is equivalent to "total absence of pain" and 10 to "greater pain bearable". This scale can be used to measure the presence and modulation of pain in the upper limb effectively [39]. A change of 1.39 points may be considered as a clinical significance [40]. A high intraclass correlation coefficient (ICC) between the NRSP and visual analog scale $(\mathrm{ICC}=0.88)$ and a good inter-rater reliability (kappa coefficient $=0.84$ ) were shown $[41,42]$.

\section{Secondary outcomes}

Both active cervical range of motion (CROM) device and Quick Disabilities of the Arm, Shoulder and Hand (DASH) scale results were established as the secondary outcomes [36]. Affected upper limb function was measured through the Quick DASH questionnaire. The Quick DASH is the abridged version of the DASH questionnaire. It is validated in Spanish and consists of 30 questions [43]. The Quick DASH questionnaire was applied only on intervention session 1 (at baseline) and 30 (at 6 weeks) [36]. Minimum clinically important difference was set at 17.1 [44]. The Quick DASH was shown to be an acceptable and valid questionnaire with low floor and ceiling effects. In addition, high internal consistency (Cronbach's a $=0.92-0.95$ ) and test-retest reliability $(\mathrm{ICC}=0.90-0.94)$ were reported $[43,45]$.

Ipsilateral cervical rotation (ICR) was assessed using a CROM device on sessions 1 (at baseline) and 30 (at 6 weeks), before and 1 hour after the application of the MNNM, DLC and OI treatments [36]. Standard error of measurement ranged from 1.6 to 2.8 degrees [46]. This measure can be used for monitoring or to assess performance during and after both conservative and invasive treatments $[47,48]$. The validity of this tool was determined by means of Pearson correlation coefficients between the CROM device and the Fastrak motion analysis system which ranged from 0.93 to 0.98 . Test-retest reliability was shown to be good (ICC $=0.89-0.98)[46,48]$.

\section{Intervention}

Participants selected in the study were stratified randomly assigned to receive one of the 3 proposed treatments. The first group ("A") received a non-invasive and non-pharmacological intervention of MNNM (Fig 1A and 1B), which was applied by a physiotherapist on a continuous basis for two minutes on five different occasions (five repetitions of continuous MNNM application) with one minute of rest between every two minutes of continuous application of the MNNM technique. The total duration of the neural mobilization application was six weeks (five interventions per week from Monday to Friday) based on a prior study which reported improvements in $\mathrm{CP}$ intensity and functionality [36]. MNNM intervention was implemented following the principles of neural mobilization established by Butler et al. [24] and Elvery \& Hall [26] for the treatment of $\mathrm{CP}$ and similar to the technique described by De la Llave et al. [16]. To begin the procedure of MNNM application, the physiotherapist placed the subject in a supine position on a stretcher where physical therapist held the patient's shoulder in $90^{\circ}$ of abduction with external rotation during the whole process of neural tissue mobilization except in the rest intervals. In order to mobilize the cervicobrachial neural tissue, an initial treatment position of the affected limb was performed, which consisted of elbow flexion with wrist and fingers extension, the subject's head was placed in a neutral position (Fig 1A). From the initial position, an elbow extension movement was performed with a wrist and finger flexion component (Fig 1B), subsequently the upper limb was immediately mobilized again, but this time the movement led to the initial position of the upper limb (flexion of elbow with extension of wrist and fingers). The participant might feel as nerve tension as reproduction of symptoms during the neural slide technique [16].

The second group (group "B") received a pharmacological treatment of IO in tablets. It was indicated by the treating physician who was familiar with the use and undesirable effects of IO on CP. The physician was in charge of modulating the doses of ibuprofen to the tolerance of the patient and in turn tried to achieve the desired hypoalgesic effect. The starting dose was a single dose of $400 \mathrm{mg} /$ day. Then the physician increased the dose linearly every day until reaching a maximum of $1200 \mathrm{mg} /$ day. The OI was divided into three doses every eight hours [31]. 

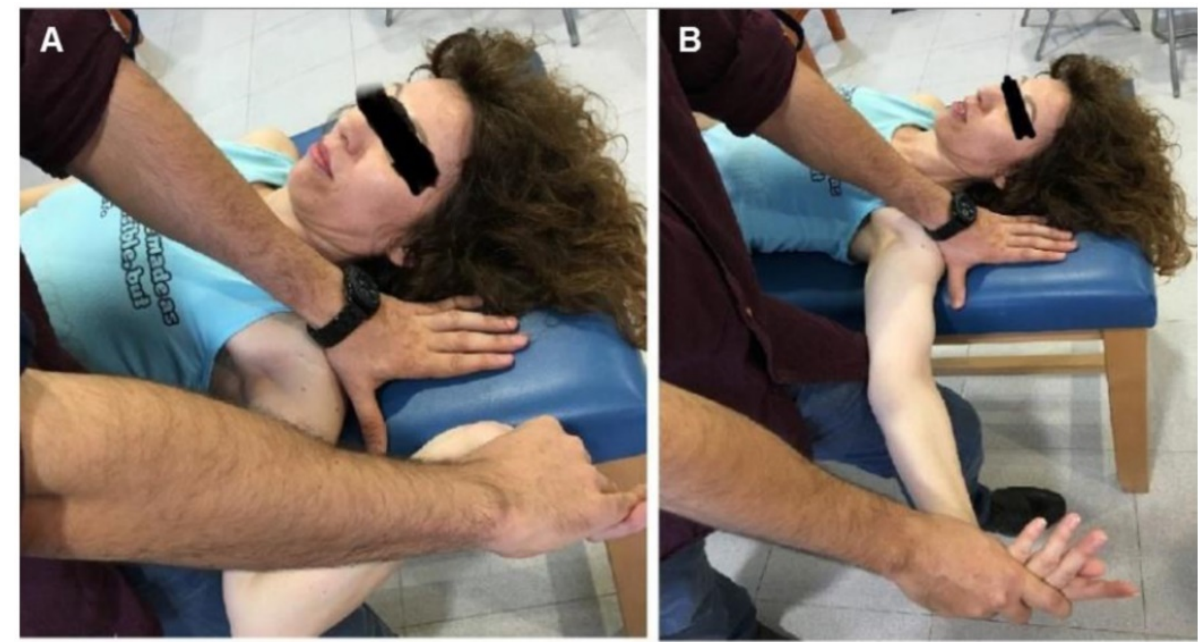

Fig 1. Initial and final positions of the MNNM slide maneuver. (A) Initial position. (B) Final position. Abbreviation: MNNM, median nerve neural mobilization.

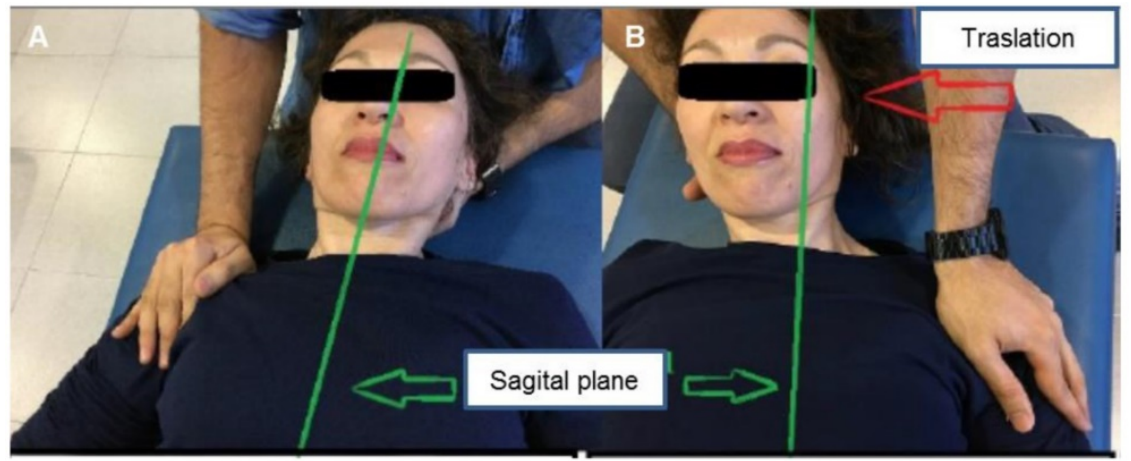

Fig 2. Initial and final positions of the CLG neural slide maneuver. (A) Initial position. (B) Final position. Abbreviation: CLG, cervical lateral glide.

Finally, the third group ("C") received a non-invasive neural mobilization treatment using the CLG technique (Fig. 2A and 2B), which was applied by a physiotherapist on a continuous basis for two minutes on five different occasions (five repetitions of continuous CLG application) with one minute of rest between every two minutes of continuous application of the CLG technique. The total duration of the CLG application was six weeks ((five interventions per week from Monday to Friday)) based on a prior study which reported improvements in $\mathrm{CP}$ intensity and functionality [36]. The CLG intervention was implemented following the principles of neural mobilization established by Butler et al. [24], Elvery \& Hall [26] for the treatment of $\mathrm{CP}$ and similar to the technique extensively described by Allison et al. [17]. The CLG technique was applied by means of an initial supine positioning of the subject on a stretcher, with both elbows in $90^{\circ}$ flexion, shoulders in slight abduction and both hands resting on the abdomen or chest (Fig 2A). The physiotherapist carefully stabilized the shoulder in the acromial region with one hand while holding the subject's neck and head. The gliding technique was performed in a controlled and careful way in a contra-lateral direction to the affected side until a point prior to the reproduction of pain or the perception of a cervical joint barrier that will block the sliding movement (Fig 2B) $[17,36]$.

\section{Sample size}

The sample size calculation method used in the present study was performed through computer software available at http://med.unne.edu.ar/ biblioteca/calculos/calculadora.htm. The estimated sample size was of 105 subjects, at a 95\% confidence level, an estimated 5\% error (with a two-tailed hypothesis), a power $(1-\beta)$ of 0.90 as well as an estimation of improvement in the $50 \%$ of the sample. The total sample of 144 participants assessed for eligibility showed similar sociodemographic data and the same diagnosis as well as were evaluated 1 week before the start of the study. Therefore, 3 groups of 35 participants were satisfactory for their comparison.

\section{Statistical method}

SPSS version 22.0 for Windows (IBM Corp. Released 2013. IBM SPSS Statistics for Windows, Version 22.0. Armonk, NY: IBM Corp) was used for statistical analysis. The statistical tests were performed considering a 95\% confidence interval $(P<$ 
.05). Only the data collected from subjects who ended the trial was processed, since they had to be evaluated in terms of pain according to the NRSP scale, the Quick DASH scale and the CROM measurement. Results were expressed in absolute frequencies, percentages, mean, standard deviation (SD) and 95\% confidence intervals. The Kolmogorov Smirnov test was used to test normality. Mean and SD were use to describe the age and outcome measurements. The gender was described by frequencies and percentages. One factor analysis of variance (ANOVA) with the Fisher's F-test was applied to test age differences between the groups. Chi square test with the $X^{2}$ statistic was applied to test gender differences between treatment groups. Repeated-measures ANOVA with 2 factors (considering the significance of the Greenhouse-Geisser correction when the Mauchly test rejected the sphericity) and Bonferroni's correction were applied to determine the intergroup comparison for $\mathrm{CP}$ intensity (3 groups $\times 6$ measurements), range of motion (3 groups $\times 4$ measurements) and physical function (3 groups $\times 2$ measurements). Furthermore, the effect size was calculated by the Eta ${ }^{2}$ coefficient. In order to simplify the exposure of the repeated measures ANOVA and Bonferroni's correction results, only the values related to the interaction of the applied treatments (MNNM and CLG versus OI) over the dependent variables (pain modulation measured through the NRSP, upper limb function measured through the Quick DASH questionnaire and ICR assessed through a CROM Device) were reported.

\section{Results}

\section{Demographic data and flow diagram}

Considering the Table 1, demographic data of the 3 groups did not show statistically significant differences for gender $\left(X^{2}=4.550 ; P=.103\right)$ or age $\left(\mathrm{F}_{(16,74)}=1.364 ; P=.192\right)$.

All subjects were Hispanics. The flow diagram is shown in Fig 3. Despite a total sample of 105 subjects was initially randomized, only 75 patients were finally analysed in the MNNM $(n=24)$, CLG $(n=25)$ and OI $(n=26)$ groups.

Table 1. Demographic data of participants according to treatment group.

\begin{tabular}{lllll}
$\begin{array}{l}\text { Demographic } \\
\text { data }\end{array}$ & $\begin{array}{l}\text { MNNM (a) } \\
\text { (n= 24) }\end{array}$ & $\begin{array}{l}\text { CLG (b) } \\
\text { (n= 25) }\end{array}$ & $\begin{array}{l}\text { OI (c) } \\
\text { (n= 26) }\end{array}$ & $\begin{array}{l}\boldsymbol{P} \text { - value } \\
\text { (Statistic) }\end{array}$ \\
\hline $\begin{array}{l}\text { Age (y) mean } \\
\pm \text { SD }\end{array}$ & $32.3 \pm 3.6$ & $33.3 \pm 5.0$ & $30.8 \pm 4.2$ & $\begin{array}{l}P=.192^{*} \\
\left(\mathrm{~F}_{(16,74)}=1.364\right)\end{array}$ \\
$\begin{array}{l}\text { Female } \\
\text { gender }\end{array}$ & $13(54.2)$ & $11(44.0)$ & $19(73.1)$ & $\begin{array}{l}P=.103^{\dagger}\left(\chi^{2}=\right. \\
\text { frequency (\%) }\end{array}$ \\
\hline
\end{tabular}

Abbreviations: CLG, cervical lateral glide; $\mathrm{MNNM}=$ median nerve neural mobilization; $\mathrm{OI}=$ oral ibuprofen

*One factor analysis of variance (ANOVA) with the Fisher's F-test was applied. tChi square test with the $\chi^{2}$ statistic was applied.

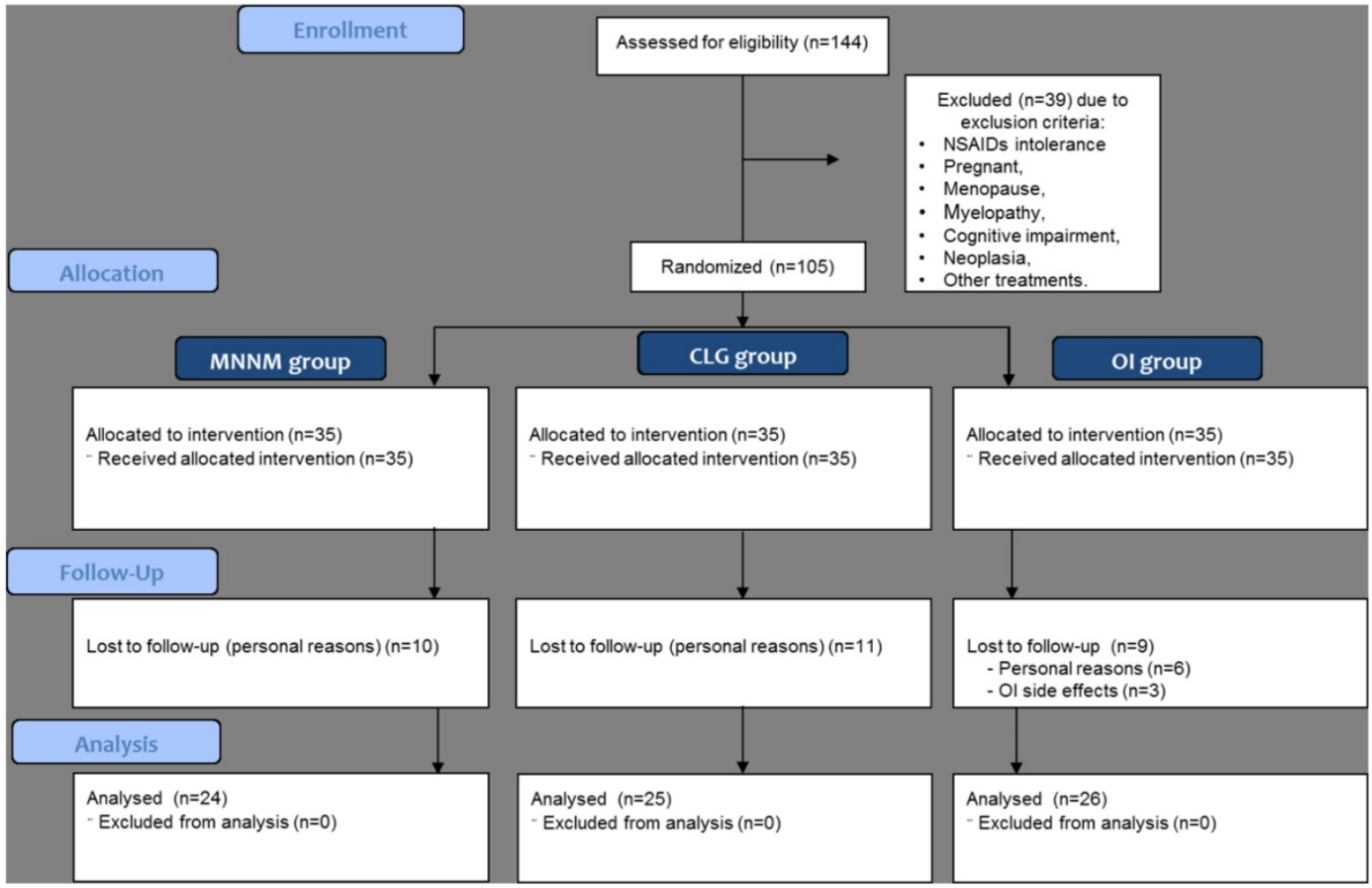

Fig 3. Participant flow through the trial. Abbreviations: CLG, cervical lateral glide; NSAIDs, non-steroidal antiinflammatory drugs; MNNM, median nerve neural mobilization; Ol, oral ibuprofen. 


\section{Pain intensity}

Intergroup statistically significant differences were shown for $\mathrm{CP}$ intensity with a large effect size $\left(\mathrm{F}_{(2,72)}=22.343 ; P<.001 ; \mathrm{Eta}^{2}=0.383\right)$. Indeed, Bonferroni's correction showed statistically significant differences $(P<.01 ; 95 \% \mathrm{CI}=0.22-3.26)$ for $\mathrm{CP}$ intensity reduction in favor of the OI treatment at all measurement moments, except for baseline comparison (Fig 4).

\section{Range of motion}

CROM did not show intergroup statistically significant difference $\left(\mathrm{F}_{(2,72)}=1.434 ; P=.245 ; \mathrm{Eta}^{2}=\right.$ 0.038) (Fig 5).

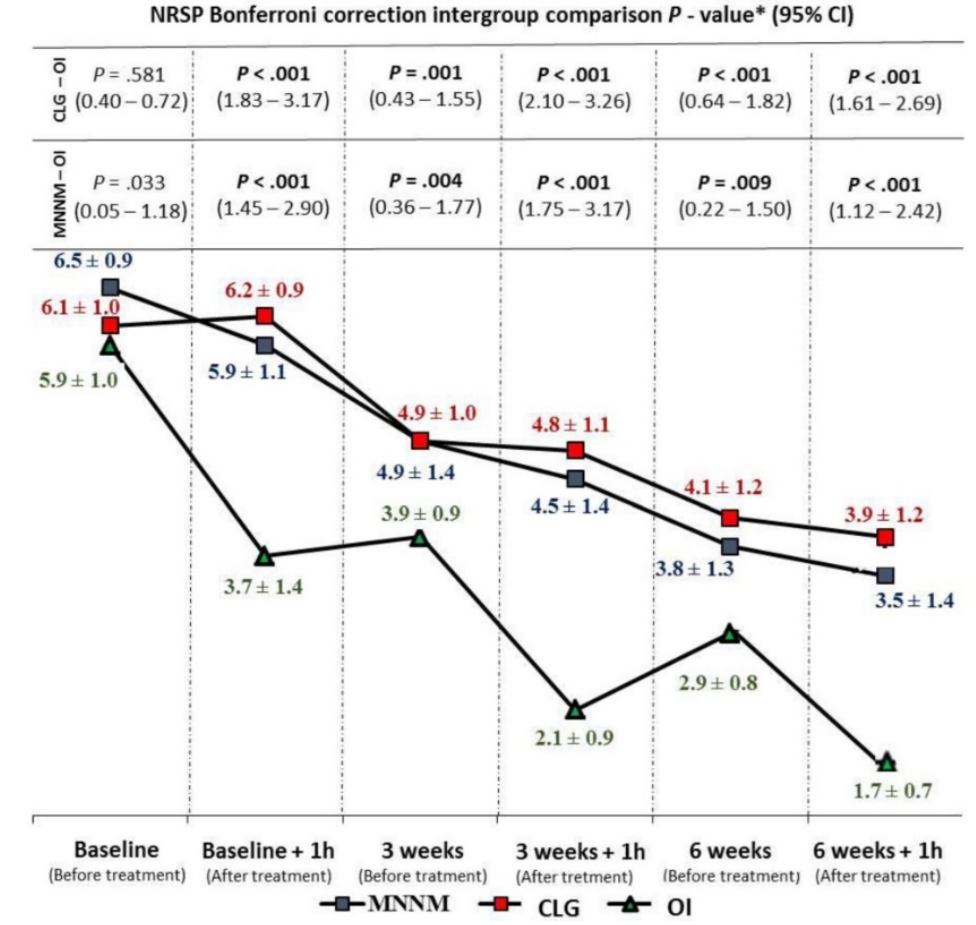

Fig 4. CP intensity intergroup comparison during follow-up. Abbreviations: CLG, cervical lateral glide; $\mathrm{Cl}$, confidence interval; h, hour; $\mathrm{MNNM}$, median nerve neural mobilization; NRSP, numeric rating scale for pain intensity; OI, oral ibuprofen. *According to the $P$-values obtained by the Bonferroni correction $(P<.05 / 3$; significance $<.017)$.

CROM Bonferroni correction intergroup comparison $P$ - value* $(95 \% \mathrm{Cl})$

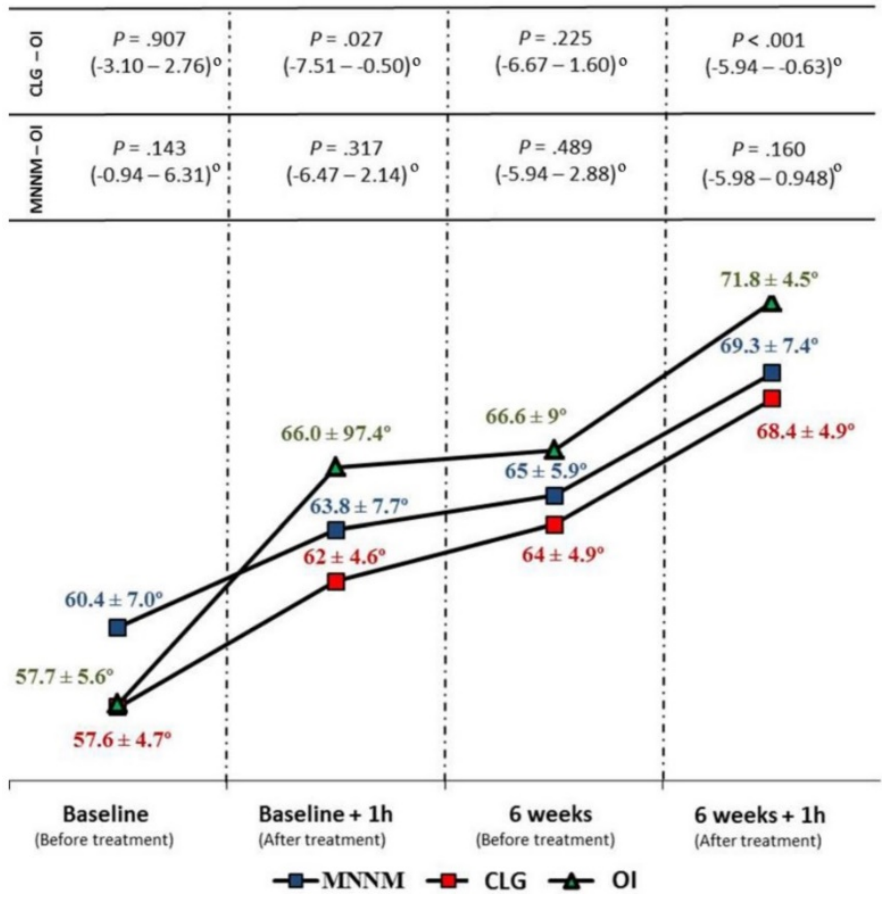

Fig 5. Range of motion intergroup comparison during follow-up. Abbreviations: $\mathrm{CLG}$, cervical lateral glide; $\mathrm{Cl}$, confidence interval; $\mathrm{CROM}$, cervical range of motion; $\mathrm{h}$, hour; MNNM, median nerve neural mobilization; OI, oral ibuprofen. *According to the $P$-values obtained by the Bonferroni correction $(P<.05 / 3$; significance $<.017)$. 
Quick DASH Bonferroni correction intergroup comparison $P$ - value* $(95 \% \mathrm{Cl})$

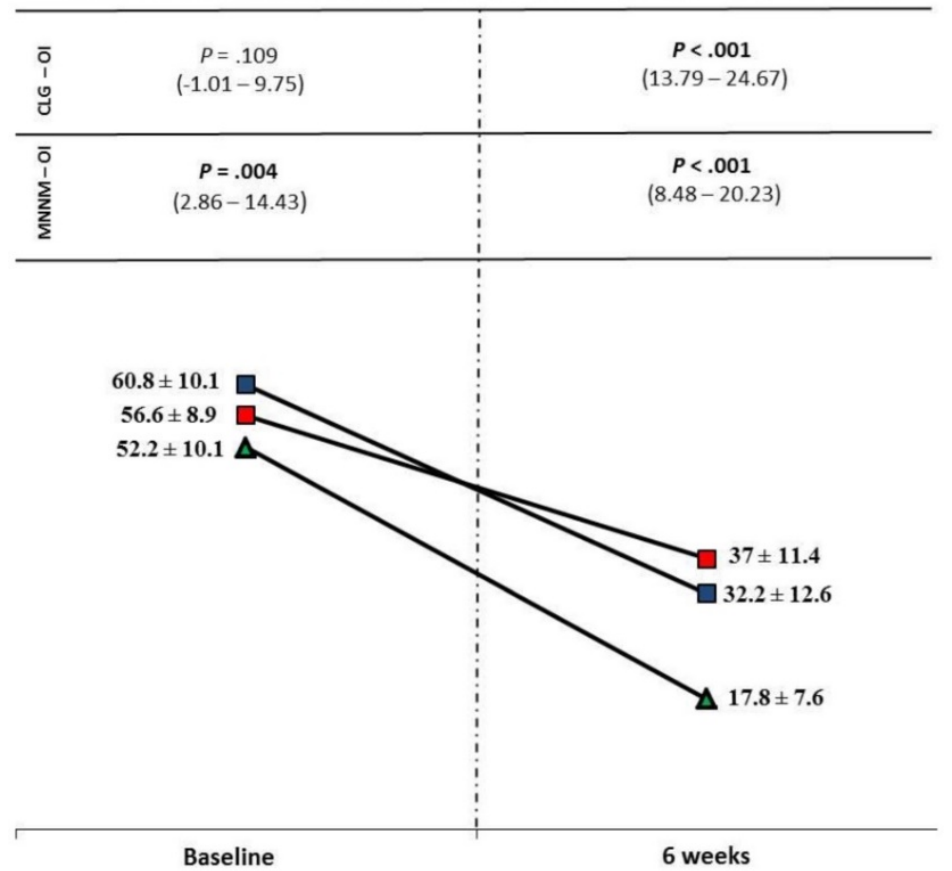

Fig 6. Physical function intergroup comparison during follow-up. Abbreviations: $\mathrm{CLG}$, cervical lateral glide; $\mathrm{Cl}$, confidence interval; h, hour; $\mathrm{MNNM}$, median nerve neural mobilization; OI, oral ibuprofen; Quick DASH, Quick Disabilities of the Arm, Shoulder and Hand. *According to the $P$-values obtained by the Bonferroni correction $(P<$ $.05 / 3$; significance <.017).

\section{Physical function}

Intergroup statistically significant differences were shown for Quick DASH with a large effect size $\left(\mathrm{F}_{(2,72)}=15.338 ; P<.001 ; \mathrm{Eta}^{2}=0.299\right)$. Indeed, Bonferroni's correction showed statistically significant differences $(P<.01 ; 95 \% \mathrm{CI}=2.86-24.67)$ for disability reduction in favor of the OI treatment at both measurement moments, except for baseline comparison between CLG and OI (Fig 6).

\section{Discussion}

This novel study provides useful findings for the conservative treatment of $\mathrm{CP}$. Despite OI may be considered a better treatment than CLG and MNNM for pain intensity and disability reduction during 6 weeks, neural mobilization showed the same ICR and may reduce the possibility of side effects $[18,29,30]$. Considering previous literature with a control group, neural mobilization was shown to be superior to the absence of treatment in reducing pain and increasing the affected upper limb function of subjects who suffer CP (36).

Regarding the clinical significance of OI versus both neural mobilizations (MNNM and CLG), the main outcome only reached the minimum clinical significance of 1.39 points for the NRSP at 1 hour after treatments, but not for the rest of primary outcome measurements (Fig 4) [40]. Nevertheless, the minimum clinically important difference of 17.1 for Quick DASH was reached between CLG and OI treatments, although not between MNNM and OI interventions (Fig 6) [44]. Considering neural mobilization treatments, Salt et al. showed that the addition of a lateral-glide mobilization to a self-management program did not produce pain intensity or functionality improvements in patients with chronic CP during 6 weeks and may result in higher health-care costs [49]. Nevertheless, CLG provided pain intensity and functionality improvements with respect to a waiting-list control of patients with CP [36]. Furthermore, Nee et al. supported that neural tissue management may provide immediate clinically relevant benefits in pain and function with no evidence of harmful effects in participants with CP [29]. During the process of results analysis a significant discrepancy was determined about the effectiveness of $\mathrm{OI}$ in the treatment of CP. Therefore, OI may not produce a significant effect over $\mathrm{CP}$, according to Sheather-Reid \& Cohen [14]. It is important to state that although OI is recommended worldwide for the treatment of $\mathrm{CP}$ [30], this discrepancy may be a direct consequence of the used $800 \mathrm{mg} /$ day dose. This dose of $800 \mathrm{mg} /$ day is considered a dosage significantly lower than the recommended $1200 \mathrm{mg} /$ day to achieve an 
appropriate analgesic-anti-inflammatory effect [50]. Despite OI may produce lower percentage of side effects than other pharmacologic treatments such as clonidine $(74 \%)$ or codeine $(69 \%)$, up to $28 \%$ of patients may suffer from sedation, dizziness, and other side effects [51].

\section{Limitations}

The main study limitations were the lack of a placebo or control group and the high loss to follow-up which provided a final sample size of 75 participants while the sample size calculation determined at least 105 participants. The retrospective trial registry should be considered, and futures studies should be registered prospectively. Furthermore, Bonferroni's correction showed statistically significant differences between MNNM and OI groups for Quick DASH scores at baseline (Fig 6). Therefore, the results of this study should be considered with caution. Indeed, side effects were not analyzed and should be considered due to the presence of adverse effects in the OI group (Fig 3) [30]. Finally, the musculoskeletal or neuropathic origin of $\mathrm{CP}$ suffered by the subjects was not established and may clearly influence the results [52]. According to Gangavelli et al., only $19.9 \%$ of CP cases may be consequence of neurogenic origin [3]. In addition, pain catastrophizing or beliefs may alter the follow-up, outcome measurements and treatments effectiveness [53,54].

\section{Conclusion}

In conclusion, OI pharmacologic treatment may reduce pain intensity and disability with respect to neural mobilization (MNNM and CLG) in patients with CP during six weeks. Nevertheless, the non-existence of between-groups ROM differences and possible OI adverse effects should be considered.

\section{Clinical Trial Registry}

The trial was registered at ClinicalTrials.gov (NCT02595294, NCT02593721).

\section{Competing Interests}

The authors have declared that no competing interest exists.

\section{References}

1. Cleland JA, Whitman JM, Fritz JM, Palmer JA. Manual Physical Therapy, Cervical Traction, and Strengthening Exercises in Patients With Cervical Radiculopathy: A Case Series. J Orthop Sport Phys Ther. 2005; 35: 802-11.

2. Radhakrishnan K, Litchy WJ, O'Fallon WM, Kurland LT. Epidemiology of cervical radiculopathy. A population-based study from Rochester, Minnesota, 1976 through 1990. Brain. 1994; 117: 325-35.

3. Gangavelli R, Nair NS, Bhat AK, Solomon JM. Cervicobrachial pain - How Often is it Neurogenic? J Clin Diagn Res. 2016; 10: YC14-6.

4. Fisher RFG, Shaw PLF. Papiro quirúrgico de Edwin Smith. Historia y Filosofía de la Medicina. A Méd. 2005; 50: 43-8.
5. Wagner SC, Van Blarcum GS, Kang DG, Lehman RA. Operative treatment of new onset radiculopathy secondary to combat injury. Mil Med. 2015; 180: 137-40.

6. Eubanks JD. Cervical radiculopathy: nonoperative management of neck pain and radicular symptoms. Am Fam Physician. 2010; 81: 33-40.

7. Peolsson A, Söderlund A, Engquist M, Lind B, Löfgren H, Vavruch L, et al. Physical Function Outcome in Cervical Radiculopathy Patients After Physiotherapy Alone Compared With Anterior Surgery Followed by Physiotherapy. Spine (Phila Pa 1976). 2013; 38: 300-7.

8. Caridi JM, Pumberger M, Hughes AP. Cervical radiculopathy: a review. HSS J. 2011; 7: 265-72.

9. Dillingham TR. Evaluating the Patient With Suspected Radiculopathy. PM\&R. 2013; 5: S41-9.

10. Boyles R, Toy P, Mellon J, Hayes M, Hammer B. Effectiveness of manual physical therapy in the treatment of cervical radiculopathy: a systematic review. J Man Manip Ther. 2011; 19: 135-42. A

11. Young IA, Michener LA, Cleland JA, Aguilera AJ, Snyder AR. Manual therapy, exercise, and traction for patients with cervical radiculopathy: a randomized clinical trial. Phys Ther. 2009; 89: 632-42.

12. Dabbs V, Lauretti WJ. A risk assessment of cervical manipulation vs. NSAIDs for the treatment of neck pain. J Manipulative Physiol Ther. 1995; 18: 530-6.

13. van der Velde G, Hogg-Johnson S, Bayoumi AM, Cassidy JD, Côté P, Boyle E, et al. Identifying the Best Treatment Among Common Nonsurgical Neck Pain Treatments. J Manipulative Physiol Ther. 2009; 32: S209-18.

14. Sheather-Reid RB, Cohen M. Efficacy of analgesics in chronic pain: a series of N-of-1 studies. J Pain Symptom Manage. 1998; 15: 244-52.

15. Day RO. Variability in response to NSAIDs: what progress? Agents Actions Suppl. 1993; 44: 3-6.

16. Isabel de-la-Llave-Rincón A, Puentedura EJ, Fernández-de-Las-Peñas C. Clinical presentation and manual therapy for upper quadrant musculoskeletal conditions. J Man Manip Ther. 2011; 19: 201-11.

17. Allison GT, Nagy BM, Hall T. A randomized clinical trial of manual therapy for cervico-brachial pain syndrome -- a pilot study. Man Ther. 2002; 7: 95-102.

18. Clark BD, Barr AE, Safadi FF, Beitman L, Al-Shatti T, Amin M, et al. Median nerve trauma in a rat model of work-related musculoskeletal disorder. J Neurotrauma. 2003; 20: 681-95.

19. Bove GM. Epi-perineurial anatomy, innervation, and axonal nociceptive mechanisms. J Bodyw Mov Ther. 2008; 12: 185-90.

20. Elvey R. Brachial plexus tension test and the pathoanatomical origin of arm pain. In: Glasgow E TL, editor. Aspects of manipulative therapy. Lincoln In. Melbourne; 1979. p. 105-110.

21. Elliott MB, Barr AE, Kietrys DM, Al-Shatti T, Amin M, Barbe MF. Peripheral neuritis and increased spinal cord neurochemicals are induced in a model of repetitive motion injury with low force and repetition exposure. Brain Res. 2008; 1218: 103-13.

22. Chu J, Allen DD, Pawlowsky S, Smoot B. Peripheral response to cervical or thoracic spinal manual therapy: an evidence-based review with meta analysis. J Man Manip Ther. 2014; 22: 220-9.

23. Kietrys DM, Palombaro KM, Azzaretto E, Hubler R, Schaller B, Schlussel JM, et al. Effectiveness of dry needling for upper-quarter myofascial pain: a systematic review and meta-analysis. J Orthop Sports Phys Ther. 2013; 43: 620-34

24. Butler DS, Coppieters MW. Neurodynamics in a broader perspective. Man Ther. 2007; 12: e7-8.

25. Coppieters MW, Butler DS. Do "sliders" slide and "tensioners" tension? An analysis of neurodynamic techniques and considerations regarding their application. Man Ther. 2008; 13: 213-21. A

26. Elvery R, Hall T. Neural tissue evaluation and treatment. 3rd ed. Donatelli R, editor. New York: Churchill Livingstone; 1997. 131-152 p.

27. Beneciuk JM, Bishop MD, George SZ. Effects of upper extremity neural mobilization on thermal pain sensitivity: a sham-controlled study in asymptomatic participants. J Orthop Sports Phys Ther. 2009; 39: 428-38.

28. Coppieters MW, Stappaerts KH, Wouters LL, Janssens K. The Immediate Effects of a Cervical Lateral Glide Treatment Technique in Patients With Neurogenic Cervicobrachial Pain. J Orthop Sport Phys Ther. 2003; 33: 369-78.

29. Nee RJ, Vicenzino B, Jull GA, Cleland JA, Coppieters MW. Neural tissue management provides immediate clinically relevant benefits without harmful effects for patients with nerve-related neck and arm pain: a randomised trial. J Physiother. 2012; 58: 23-31.

30. Rainsford KD. Ibuprofen: pharmacology, efficacy and safety. Inflammopharmacology. 2009; 17: 275-342.

31. Rainsford KD. Ibuprofen: pharmacology, efficacy and safety. Inflammopharmacology. 2009; 17: 275-342.

32. Carlesso LC, Macdermid JC, Gross AR, Walton DM, Santaguida PL. Treatment preferences amongst physical therapists and chiropractors for the management of neck pain: results of an international survey. Chiropr Man Therap. 2014;22:11.

33. Boutron I, Dutton S, Ravaud P, Altman DG. Reporting and Interpretation of Randomized Controlled Trials With Statistically Nonsignificant Results for Primary Outcomes. JAMA. 2010; 303: 2058

34. Bono CM, Ghiselli G, Gilbert TJ, Kreiner DS, Reitman C, Summers JT, et al. An evidence-based clinical guideline for the diagnosis and treatment of cervical radiculopathy from degenerative disorders. Spine J. 2011;11:64-72.

35. Weiner DK, Ernst E. Complementary and alternative approaches to the treatment of persistent musculoskeletal pain. Clin J Pain. 2004;20:244-55. 
36. Rodríguez-Sanz D, Calvo-Lobo C, Unda-Solano F, Sanz-Corbalán I, Romero-Morales C, López-López D. Cervical Lateral Glide Neural Mobilization Is Effective in Treating Cervicobrachial Pain: A Randomized Waiting List Controlled Clinical Trial. Pain Med. 2017; 18: 2492-2503.

37. Gómez-Pérez L, López-Martínez AE, Ruiz-Párraga GT. Psychometric Properties of the Spanish Version of the Tampa Scale for Kinesiophobia (TSK). J Pain. 2011; 12: 425-35.

38. Childs JD, Piva SR, Fritz JM. Responsiveness of the numeric pain rating scale in patients with low back pain. Spine (Phila Pa 1976). 2005; 30: 1331-4.

39. MacDermid JC, Walton DM, Côté P, Santaguida PL, Gross A, Carlesso L, et al. Use of Outcome Measures in Managing Neck Pain: An International Multidisciplinary Survey. Open Orthop J [Internet]. 2013; 7: 506-20.

40. Kendrick DB, Strout TD. The minimum clinically significant difference in patient-assigned numeric scores for pain. Am J Emerg Med. 2005; 23: 828-32.

41. Hjermstad MJ, Fayers PM, Haugen DF, Caraceni A, Hanks GW, Loge JH, et al. Studies comparing Numerical Rating Scales, Verbal Rating Scales, and Visual Analogue Scales for assessment of pain intensity in adults: a systematic literature review. J Pain Symptom Manage. 2011; 41: 1073-93.

42. Basson CA, Stewart A, Mudzi W. The effect of neural mobilisation on cervico-brachial pain: design of a randomised controlled trial. BMC Musculoskelet Disord. 2014; 15: 419.

43. Angst F, Schwyzer H-K, Aeschlimann A, Simmen BR, Goldhahn J. Measures of adult shoulder function: Disabilities of the Arm, Shoulder, and Hand Questionnaire (DASH) and Its Short Version (QuickDASH), Shoulder Pain and Disability Index (SPADI), American Shoulder and Elbow Surgeons (ASES) Society Standardized Shoulder. Arthritis Care Res (Hoboken). 2011; 63(S11): S174-88.

44. van Kampen DA, Willems WJ, van Beers LWAH, Castelein RM, Scholtes VAB, Terwee CB. Determination and comparison of the smallest detectable change (SDC) and the minimal important change (MIC) of four-shoulder patient-reported outcome measures (PROMs). J Orthop Surg Res. 2013 N; 8: 40 .

45. Gummesson C, Ward MM, Atroshi I. The shortened disabilities of the arm, shoulder and hand questionnaire (QuickDASH): validity and reliability based on responses within the full-length DASH. BMC Musculoskelet Disord. 2006; 7: 44 .

46. Audette I, Dumas J-P, Côté JN, De Serres SJ. Validity and between-day reliability of the cervical range of motion (CROM) device. J Orthop Sports Phys Ther. 2010; 40: 318-23.

47. Youdas JW, Carey JR, Garrett TR. Reliability of measurements of cervical spine range of motion--comparison of three methods. Phys Ther. 1991; 71: 98-104-6.

48. Fletcher JP, Bandy WD. Intrarater reliability of CROM measurement of cervical spine active range of motion in persons with and without neck pain. J Orthop Sports Phys Ther. 2008; 38: 640-5.

49. Salt E, Kelly $\mathrm{S}$, Soundy A. Randomised Controlled Trial for the Efficacy of Cervical Lateral Glide Mobilisation in the Management of Cervicobrachial Pain. Open J Ther Rehabil. Scientific Research Publishing; 2016;4(3):132-45.

50. McQuay HI, Moore RA. Dose-response in direct comparisons of different doses of aspirin, ibuprofen and paracetamol (acetaminophen) in analgesic studies. Br J Clin Pharmacol. 2007; 63: 271-8.

51. Max MB, Schafer SC, Culnane M, Dubner R, Gracely RH. Association of pain relief with drug side effects in postherpetic neuralgia: A single-dose study of clonidine, codeine, ibuprofen, and placebo. Clin Pharmacol Ther. 1988; 43: 363-71.

52. Moloney N, Hall T, Doody C. An investigation of somatosensory profiles in work related upper limb disorders: a case-control observational study protocol. BMC Musculoskelet Disord. 2010; 11: 22.

53. Lopez-de-Uralde-Villanueva I, Beltran-Alacreu H, Fernandez-Carnero J, Kindelan-Calvo P, La Touche R. Widespread Pressure Pain Hyperalgesia in Chronic Nonspecific Neck Pain with Neuropathic Features: A Descriptive Cross-Sectional Study. Pain Physician. 2016; 19 :77-88.

54. Beltran-Alacreu H, López-de-Uralde-Villanueva I, Fernández-Carnero J, La Touche R. Manual Therapy, Therapeutic Patient Education, and Therapeutic Exercise, an Effective Multimodal Treatment of Nonspecific Chronic Neck Pain. Am J Phys Med Rehabil. 2015; 94: 887-97. 